\title{
ESTUDIO DE PREFACTIBILIDAD PARA LA ELABORACIÓN DE MUEBLES DE FIBRA DE CASHAVARA (Desmoncus polyacanthos) CON FINES DE EXPORTACIÓN
}

\author{
Badys CHUQUIZUTA DEL CASTILLO', Ximena TAGLE CASAPÍA², José L. RODRIGUEZ GÓMEZ1, \\ Dennis DEL CASTILLO TORRES ${ }^{2}$ \\ 1 Universidad Nacional de la Amazonía Peruana, Sargento Lores 385, Iquitos, Perú. E-mail: badys92@hotmail.com \\ 2 Instituto de Investigaciones de la Amazonía Peruana, IIAP. Av. Abelardo Quiñones Km 2.5, Iquitos, Perú. E-mail: \\ xtagle@iiap.org.pe
}

\begin{abstract}
RESUMEN
Los productos forestales no maderables están cobrado notable auge. A nivel global, existe una demanda creciente en el uso de materias primas renovables, y en particular, en el uso de muebles fabricados con fibras naturales. La demanda de estos productos es dominada principalmente por el mercado de Estados Unidos con una participación del 28\%. En la Amazonía peruana, la cashavara (Desmoncus polyacanthos) es una palmera trepadora que provee de fibras de características similares a las del ratán y que son utilizadas en forma de tejidos denominados "esterillados" en la elaboración de muebles. El presente estudio evaluó a nivel de prefactibilidad, la viabilidad técnica de la transformación y la comercialización de muebles con esterillado de fibra de cashavara al mercado estadounidense. Para ello, se realizó un estudio de mercado y un análisis técnico, donde se determinó que existe una demanda insatisfecha, la cual podría ser cubierta en un $0.46 \%$ produciendo 300 muebles anuales. La evaluación económica-financiera del proyecto se estimó en base a los indicadores de rentabilidad. Concluimos que el proyecto es rentable al tener un VAN de USD 183423.56 y TIR de 55\%. Asimismo, se determinó que por cada US dólar invertido, se reintegran USD 1.23. Es importante mencionar que el estudio consideró la resistencia a ciertos cambios que puedan sufrir variables como precio de venta y precio de materia prima.
\end{abstract}

PALABRAS CLAVE: prefactibilidad, estudio de mercado, fibra, cashavara, Desmoncus polyacanthos.

\section{FEASIBILITY STUDY FOR THE DEVELOPMENT OF FURNITURE USING CASHAVARA (Desmoncus polyacanthos) FIBER FOR EXPORT ATION}

\begin{abstract}
The non-timber forest products have gained remarkable interest. Globally, there is increasing demand in the use of renewable raw materials, and in particular the use of furniture made of natural fibers. Demand for these products is mainly dominated by the US market with a share of $28 \%$. In the Peruvian Amazon, the cashavara (Desmoncus polyacanthos) is a climbing palm that provides fibers with similar characteristics to rattan, and it is used in making furniture with canework (woven fiber). This study aimed to assess the feasibility level of processing and marketing furniture with cashavara fibers to the US market. For this, we developed a market assessment and technical analysis to determine if there is an unmet demand, which could be covered in $0.46 \%$ producing 300 furniture annually. The economic-financial evaluation of the project was estimated based on profitability indicators. We conclude that the project is profitable when the NPV is USD 183423.56 and the IRR is 55\%. It was also found that for every US dollar invested USD 1.23 was reintegrated. It is noteworthy that the study considered resistance to certain changes that may suffer variables as selling price and raw material price.
\end{abstract}

KEYWORDS: feasibility, market assessment, fiber, cashavara, Desmoncus polyacanthos. 


\section{INTRODUCCIÓN}

Los productos forestales no maderables cumplen un rol importante en la vida del poblador amazónico al brindar opciones adicionales para el desarrollo sostenible, generando empleo e ingresos económicos (Baluarte, 2000; Meza et al., 2006). En este contexto, las fibras naturales son uno de los productos con mayor potencial de comercialización. Especialmente aquellas empleadas en la fabricación de muebles, debido al incremento global de la demanda de materias primas renovables.

El mercado de las fibras naturales actualmente está dominado por el comercio del "ratán" (Calamus spp.), "mimbre" (Heteropsis oblongifolia), "abacá" (Musa textilis) y "rafia" (Rhapis excelsa). Siendo los principales países exportadores China, Italia, Polonia y Estados Unidos. Perú participa con una cuota mínima de "mimbre" y recientemente con "cashavara" (Desmoncus polyacanthos Martius), esta última especie posee propiedades similares al ratán y es utilizada para elaborar muebles con esterillados (Isnard et al., 2005; Balsev et al., 2008; Guevara et al., 2011).

Estudios de prefactibilidad con fibras naturales para la elaboración de muebles en Ecuador demuestran la viabilidad de producción y exportación en países Latinoamericanos. Un estudio realizado por Intriago et al. (2009) señala que es factible producir muebles de abacá pues existe una demanda, la maquinaria adecuada y las herramientas necesarias para su fabricación en ese país. El análisis financiero concluyó que este proyecto tiene un buen nivel de rentabilidad del $22 \%$, porcentaje mayor a la tasa de descuento del $12 \%$, considerándose atractivo. El VAN del proyecto refleja un resultado positivo en $70 \%$ mientras que sólo existe un $30 \%$ de riesgo.

Asimismo, un estudio de pre-factibilidad realizado por Plazarte (2010) para la exportación de muebles de fibra de mimbre a Canadá concluyó que este es un mercado muy atractivo, debido a que Canadá es un país que está en busca de "muebles verdes", es decir, aquellos que tienen menor impacto ambiental. El rendimiento obtenido en ese proyecto es superior al porcentaje mínimo exigido por la tasa de descuento, ya que ésta exige un rendimiento del $13.78 \%$ para el proyecto y en la TIR se aprecia que se obtendría el $18 \%$, es decir 4 puntos sobre lo requerido. Adicionalmente, el VAN obtenido indicó que se acumularían USD 3263.90 en valores presentes, al recuperar la inversión, que según el PRI sería a los 10 años.

Lamentablemente en el Perú los estudios de prefactibilidad para productos forestales no maderables son escasos. Existen algunos estudios relacionados a productos alimenticios, como el de Llacsahuanga et al. (2011) para la exportación de goma de zapote (Capparis scabrida) al mercado Europeo; o estudios que presentan el potencial de comercialización de fibras naturales como Vásquez (1994) y Vela (2013). Sin embargo hay un vacío existente con respecto a estudios de prefactibilidad para la comercialización de muebles con fibras naturales, principalmente con fines de exportación.

De esta manera, el presente trabajo buscó determinar la pre-factibilidad de la organización de un grupo de trabajo de artesanos para la elaboración de muebles con esterillados de fibra de cashavara (Desmoncus polyacanthos) con fines de exportación al mercado Estadounidense, y de esta forma mejorar la calidad de vida de los pobladores del distrito de Jenaro Herrera.

\section{MATERIAL Y MÉTODOS}

\section{ÁREA DEESTUDIO}

El estudio se realizó a $200 \mathrm{~km}$ al sur de Iquitos, en el distrito de Jenaro Herrera, provincia de Requena, departamento de Loreto, entre Enero y Diciembre del 2015. El distrito de Jenaro Herrera cuenta con una población de 5507 correspondiente a trece centros poblados (INEI, 2009). La zona se caracteriza por tener extensos bosques primarios tanto de altura como inundables. El clima es cálido y húmedo con lluvias regulares durante los meses de octubre a mayo. La precipitación promedio anual es de $2730 \mathrm{~mm}$ según la estación meteorológica del Centro de Investigaciones Jenaro Herrera, para un período de observación de 24 años (1972-1994). La creciente máxima del Río Ucayali ocurre entre los meses de abril; y el nivel más bajo ocurre entre septiembre. Según CONAM (2005), la temperatura promedio es de $27^{\circ} \mathrm{C}$, con un rango entre $21^{\circ} \mathrm{C}$ y 32 ${ }^{\circ} \mathrm{C}$. El mes más caliente es noviembre. La humedad relativa promedio mensual fluctúa entre $82 \%$ (octubre) y $90 \%$ (mayo).

\section{LA ESPECIE DE INTERÉS}

La "cashavara" o "vara casha" (Desmoncus polyacanthos Martius) es una palmera trepadora que puede tener más de 40 estípites espinosos, largos y flexibles. Estos suelen ocupar el estrato medio del bosque, alcanzando longitudes de $13 \mathrm{~m}$ y diámetros de $1.3 \mathrm{~cm}$ por estípite. Presenta hojas de $20-25 \mathrm{~cm}$ de largo, con presencia de cirro (raquis foliar prolongado hacia el ápice) y una inflorescencia hermafrodita ramificada. Su fruto es oblongo a ovoide, de color amarillo a rojo cuando está maduro (Carneiro, 2006; Guevara, 2008; Torres, 2009). 
Los primeros estudios sobre su ecología en poblaciones silvestres indican que esta especie ocurre en áreas inundables del llano de la Amazonía Peruana, principalmente en restingas altas y restingas bajas (Torres, 2009; Isaza, 2013). La especie tiene un crecimiento promedio anual de 1.5 m y se distribuye ampliamente en América Central y América del Sur, desde México hasta Brasil y Bolivia; y ocurre también en el caribe a excepción de Trinidad y Tobago (Henderson et al., 1995; Troy et al., 1997; Isnard, 2005; Hubschmann et al., 2007).

La parte útil de la cashavara es el estípite o tallo, el cual llega a la madurez cuando cambia de color verde a marrón, y la corteza se empieza a desprender. La extracción de la fibra consiste en cortar el estípite en la base y jalar hasta dejarlo caer al suelo. Posteriormente se separa la parte aprovechable (desde la base hasta la parte madura) y se retira la corteza exterior (Torres, 2009; Tagle, 2014). En una cosecha de cashavara generalmente se colectan de 10 a $25 \mathrm{~kg}$ de tallos frescos, lo cual en promedio corresponde a 4-8 kg de tallos secos (Tagle, 2014). Los productores generalmente cortan los tallos por la mitad y los enrollan en madejas de aproximadamente $500 \mathrm{~g}$, las cuales miden entre 5-8 m de largo (Henderson et al,. 1993; Hübschmann et al., 2007; Guevara et al. 2011).

\section{PROCESAMIENTO Y ANÁLISIS DE LOS DATOS}

Para realizar el estudio de prefactibilidad del comercio de la cashavara, primero se realizó un estudio de mercado para la comercialización de muebles con fibras naturales a los Estados Unidos. El estudio de mercado consistió en recopilar, analizar e interpretar la información del mercado, teniendo conocimiento del rendimiento de la producción del producto, las importaciones $\mathrm{y}$ exportaciones. El rendimiento de la producción de los esterillados fue determinado a través de entrevistas semiestructuradas a los artesanos sobre la producción y comercialización de los productos. Estas entrevistas se realizaron a 12 artesanos de Jenaro Herrera y a 3 artesanos en Iquitos. Adicionalmente, usando la herramienta online Trademap (ITC, 2015), se recopiló información sobre las importaciones y exportaciones de muebles con fibras naturales a nivel mundial.

Conociendo el potencial de la venta del producto (identificación y elección del mercado objetivo, segmentación del mercado y análisis de la demanda objetivo), se procedió a evaluar técnica, financiera y económicamente el proyecto. El análisis técnico evaluó el tamaño del proyecto en función a la capacidad de producción de muebles de cashavara considerando la demanda del producto, los suministros o insumos, y el financiamiento disponible. Para ello se calculó la demanda insatisfecha de muebles en los EE. UU. y se proyectó la demanda para los próximos 10 años (cuánto compra y compraría EE. UU.), luego se procedió a determinar el porcentaje de la demanda que se cubriría según la capacidad de oferta de los productores de muebles. El tamaño del proyecto se expresa en número de juegos de muebles producidos por unidad de tiempo.

El análisis financiero consideró la infraestructura, equipos, tamaño del proyecto y todos los requerimientos del mismo; con el fin de determinar el valor total necesario de la inversión para iniciar la producción de muebles. En este proyecto, estas variables fueron evaluadas de la siguiente manera: Se obtuvo la suma de los activos fijos tangibles, activos fijos intangibles y el capital de trabajo determinando las fuentes de capital para cubrir la inversión que son cubiertas con recursos propios y recursos ajenos (crédito).

El análisis económico evaluó los costos unitarios e ingresos. Para el cálculo del costo unitario se consideraron los costos totales, divididos por la oferta anual del proyecto. La estimación de ingresos se realizó mediante la elaboración de un presupuesto que proyectó los ingresos que la empresa produciría en cierto periodo de tiempo (10 años). Para proyectar los ingresos se emplearon las unidades a vender del análisis financiero y el precio de los productos del estudio de mercado. En este estudio se consideró la tasa de inflación de Estados Unidos de 1.6\% del año 2014.

La información obtenida sirvió para realizar la evaluación financiera, donde se calcularon los indicadores de rentabilidad, tales como, valor actual neto (VAN) y tasa interna de retorno (TIR). El VAN fue estimado con la ecuación:

$$
V A N=\sum_{t=1}^{n} \frac{V_{t}}{(1+k)^{t}}-I_{0}
$$

Donde $V_{t}$ representa los flujos de caja en cada periodo $\mathrm{t}$ ( 1 año), $\mathrm{I}_{0}$ es el valor de desembolso inicial de la inversión, $\mathrm{n}$ es el número de periodos considerado (10 años), y k es el tipo de interés. Los resultados fueron evaluados siguiendo las reglas de decisión que indican una situación de indiferencia en relación al costo de oportunidad cuando el $\mathrm{VAN}=0$; se sebe rechazar el proyecto cuando las utilidades generadas no cubren los costos de oportunidad y el VAN $<0$; se debe aceptar el proyecto cuando las utilidades percibidas son mayores al costo de oportunidad y el VAN $>0$. 
La TIR fue estimada con la ecuación:

$$
T I R=\mathrm{T}_{m}+\left(\begin{array}{ll}
T M & T_{m}
\end{array}\right) \times\left[\frac{V A N T_{m}}{V A N T_{m}-V A N T M}\right]
$$

Donde Tm es la tasa de actualización menor y TM es la tasa de actualización mayor. Los resultados fueron evaluados siguiendo las reglas de decisión que indican una situación de indiferencia en relación al costo de oportunidad (COP) cuando la TIR = $\mathrm{COP}$; se debe rechazar el proyecto cuando la TIR $<$ COP; y se debe aceptar el proyecto cuando la TIR > COP. El costo de oportunidad o tasa de descuento es el precio que se paga por los fondos requeridos para cubrir la inversión y representa la medida de la rentabilidad que se exige al proyecto, según el riesgo, de tal manera que el retorno esperado permita cubrir la totalidad de la inversión inicial, los egresos de la operación, los intereses por el préstamo y la rentabilidad que el inversionista le exige a su propio capital invertido. El COP fue calculado con la ecuación $i=C R P+C R A J+T L R+$ $t i$, para el inversionista e $i=(T P \times 100 \%)+T L R+t i$, para el proyecto, donde CRP es el costo de los recursos propios, CRAJ el costo de los recursos ajenos, TLR es la tasa libre de riesgo y ti es la tasa de inflación local.

\section{RESULTADOS}

\section{ESTUDIO DE MERCADO}

La información recopilada de Trademap (2015), para el año 2014, muestra que las importaciones de muebles de fibras naturales estuvieron dominadas en un $42 \%$ por Estados Unidos, $32 \%$ por el mercado europeo y en un $26 \%$ por el mercado asiático (Figura 1). Dentro del mercado europeo los países importadores de muebles con fibras naturales son Francia, Alemania, Suiza, Reino Unido, Italia y España. En el mercado asiático, los mayores importadores de muebles con fibras naturales fueron Emiratos Árabes Unidos, India, Viet Nam, Corea, Irán, China, Rusia y Singapur.

El precio de los muebles con esterillados de fibras naturales tuvo un crecimiento de $38.9 \%$ durante el periodo 2003-2014. Aumentando significativamente cada año, desde US\$ 2701 en el 2003 hasta US\$ 3752 en el 2014 (Trademap, 2015). La tabla 1 presenta los precios en dólares valor FOB de los muebles con esterillado de fibra producidos en China, Viet Nam, Canadá, México, Malasia e Italia, los cuales proveen el $82 \%$ de sus exportaciones al mercado estadounidense. En todos ellos, el precio FOB tuvo un incremento significativo hasta el año 2009, donde luego decayó debido a la crisis económica. Sin embargo, a la fecha, los precios tienden al alza. Con respecto al precio unitario de los muebles, se estima una proyección promedio de 4617.4 miles de dólares.

\section{ANÁLISIS TÉCNICO}

El estudio de mercado demostró la existencia de una demanda insatisfecha, la cual sería cubierta en un $0.46 \%$ por la asociación de artesanos produciendo 25 juegos de sala de cashavara mensuales o 300 juegos de sala anualmente.

Para el presente estudio se compararon diferentes opciones de proveedores a lo largo de la cadena de producción, con respecto al abastecimiento de insumos, se priorizó la fluidez y calidad antes que cantidad. Seleccionamos a la provincia de Requena como principal proveedor de fibra y productor de muebles tejidos de cashavara.

Las operaciones se iniciarían en el distrito de Jenaro Herrera donde la producción de muebles se llevaría a cabo según el diseño solicitado por el cliente, para esto el bróker deberá haber fijado al cliente en el estado de Miami. Para la provisión de material de embalaje y empaque (para la protección de los muebles), se consideró trabajar con cartoneras ubicadas en la provincia de Iquitos.

Los muebles embalados serían trasladados por vía fluvial a la ciudad de Pucallpa y de allí partirían hasta el puerto del Callao para su posterior envío marítimo hasta un puerto en el estado de Miami en EE.UU.

El proyecto podría ser financiado a través de dos fuentes: capital propio y crédito bancario. El Perú cuenta con líneas de crédito otorgadas por distintas instituciones financieras.

\section{ANÁLISIS FINANCIERO}

Se determinó el valor total necesario para iniciar la producción, el cual asciende a USD 85160. Para facilitar la inversión, se consideró que el $28.84 \%$ se financiará con recursos propios y el $71.16 \%$ con recursos externos. La tabla 2 presenta los montos correspondientes a cada tipo de inversión y tipo de financiamiento, siendo los activos fijos tangibles el $80 \%$ del valor total necesario, seguido del capital de trabajo con el $17 \%$ y el $3 \%$ compuesto de activos fijos intangibles.

\section{ANÁLISIS ECONÓMICO}

El poblador local tendría que cubrir 556 soles para comercializar un juego de sala, sin obtener utilidad o créditos por ejecutar el proyecto (Tabla 3 ). Los ingresos anuales de la venta de muebles de cashavara se incrementarían con el transcurso del 
tiempo, pasando de 225000 soles a 257400 soles en 10 años de duración del proyecto (Figura 2). El precio de venta inicial sería de USD 750 por unidad, cubriendo los costos de envío y la ganancia.

\section{EVALUACIÓN FINANCIERA}

El valor actual neto (VAN), al ejecutar el proyecto, será de USD 183423.56 luego de recuperar los recursos invertidos, en un plazo de 10 años, en términos de valor presente; indicando que el proyecto es factible.

La tasa interna de retorno (TIR) obtenida es de 0.55 , lo que significa que el inversionista obtendrá el $55 \%$ de rentabilidad por cada dólar invertido de sus recursos en el proyecto, lo cual es beneficioso pues este porcentaje es mayor al que exige la tasa de descuento. Asimismo, se determinó que en tres años se recuperaría la inversión del proyecto (Tabla 4).

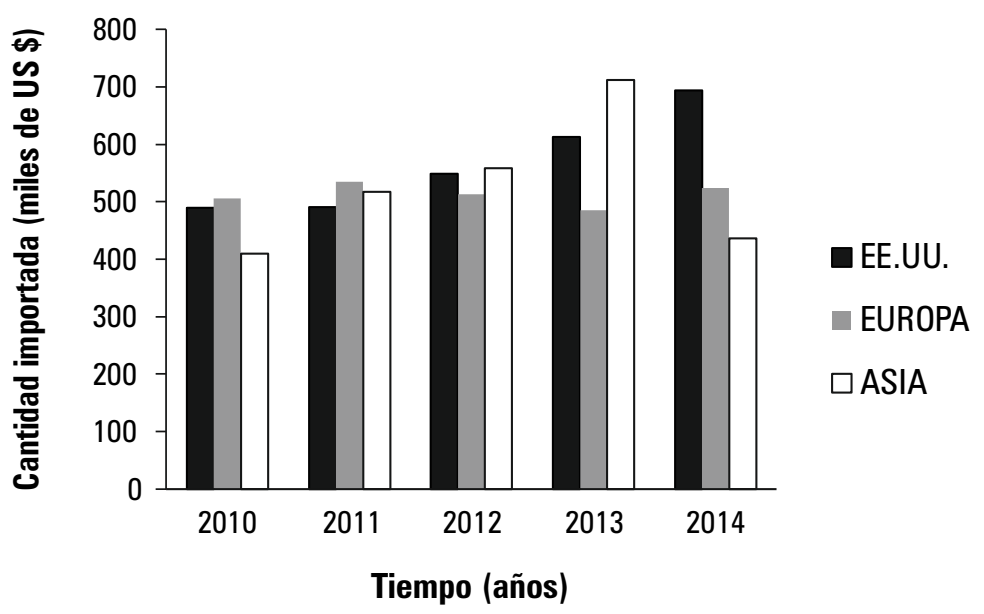

Figura 1. Importaciones totales de muebles con fibras naturales de los principales mercados internacional registradas en TRADEMAP (2014) para el periodo 2010-2014

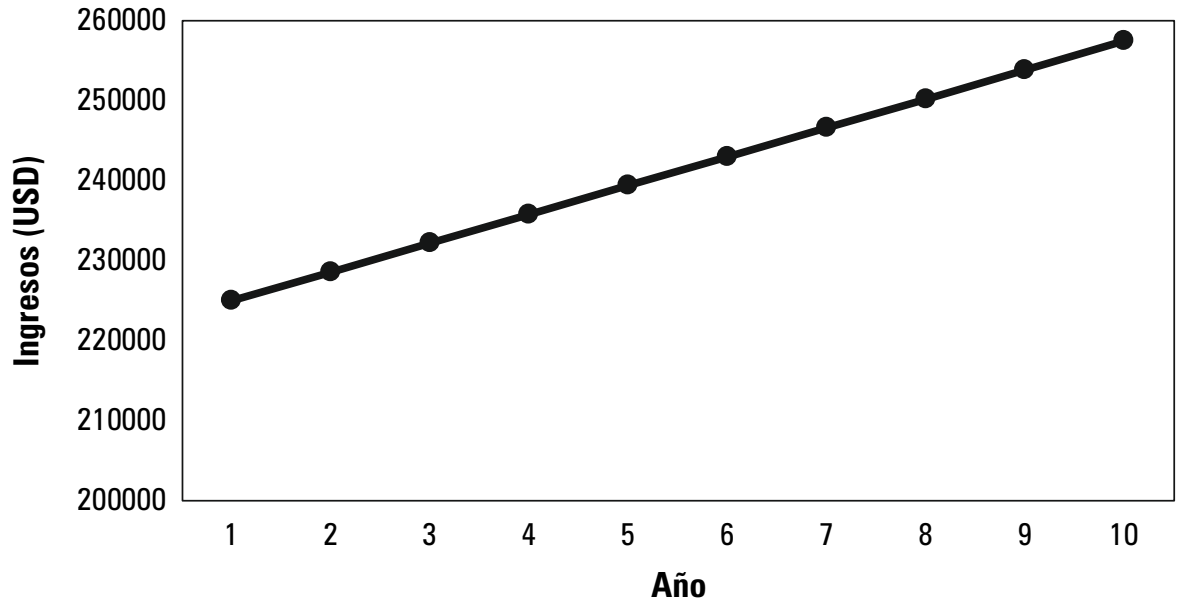

Figura 2. Ingresos anuales proyectados para 10 años de duración del proyecto de venta de muebles de cashavara por la asociación de artesanos de Jenaro Herrera a los Estados Unidos. 
Tabla 1. Evolución de los precios FOB US \$/Tm de muebles con esterillado exportados a los Estados Unidos.

\begin{tabular}{lccccccccccc}
\hline \multicolumn{1}{c}{ País } & $\mathbf{2 0 0 3}$ & $\mathbf{2 0 0 4}$ & $\mathbf{2 0 0 5}$ & $\mathbf{2 0 0 6}$ & $\mathbf{2 0 0 8}$ & $\mathbf{2 0 0 9}$ & $\mathbf{2 0 1 0}$ & $\mathbf{2 0 1 1}$ & $\mathbf{2 0 1 2}$ & $\mathbf{2 0 1 3}$ & $\mathbf{2 0 1 4}$ \\
\hline China & 2736 & 3015 & 3019 & 3023 & 3884 & 4534 & 3945 & 3813 & 4156 & 4195 & 4267 \\
Viet Nam & 2593 & 2696 & 2619 & 2629 & 3077 & 3479 & 2964 & 2864 & 3056 & 3069 & 3084 \\
Canadá & 2778 & 3023 & 3038 & 3059 & 4152 & 4737 & 4018 & 3896 & 4178 & 4154 & 4249 \\
México & 2754 & 3070 & 3070 & 3087 & 4014 & 4609 & 3986 & 3794 & 4084 & 4055 & 4091 \\
Malasia & 2586 & 2788 & 2734 & 2755 & 3313 & 3655 & 3078 & 2929 & 3089 & 3082 & 3039 \\
Italia & 2648 & 2908 & 2948 & 3009 & 3944 & 4416 & 3732 & 3508 & 3737 & 3723 & 3771 \\
\hline Total & 16095 & 17500 & 17428 & 17562 & 22384 & 25430 & 21723 & 20804 & 22300 & 22278 & 22501 \\
\hline Promedio & 2683 & 2917 & 2901 & 2927 & 3731 & 4238 & 3620 & 3467 & 3716 & 3713 & 3750 \\
\hline
\end{tabular}

Tabla 2. Descripción de los montos a invertir según fuente de financiamiento para el inicio del proyecto de venta de muebles de cashavara por los pobladores de Jenaro Herrera a los Estados Unidos.

\begin{tabular}{l|c|c|c|c|c}
\hline \multirow{2}{*}{ Descripción } & \multirow{2}{*}{$\begin{array}{c}\text { Valor } \\
\text { (USD) }\end{array}$} & \multicolumn{2}{c|}{ Recursos propios } & \multicolumn{2}{c}{ Recursos ajenos } \\
\cline { 3 - 6 } & & $\%$ & USD & $\%$ & USD \\
\hline Edifico & 17754.51 & 0 & 0 & 100 & 17754.51 \\
\hline Terreno & 7910.43 & 0 & 0 & 100 & 7910.43 \\
Vehículo & 33868.34 & 0 & 0 & 100 & 33868.34 \\
Maquinaria y equipo taller & 3867.01 & 100 & 3867.01 & & 0.00 \\
Maquinaria y equipo bodega & 292.98 & 100 & 292.98 & & 0.00 \\
Muebles y enseres & 1439.11 & 100 & 1439.11 & & 0.00 \\
Equipos de computación & 2139.20 & 50 & 1069.60 & 50 & 1069.60 \\
\hline Equipos de oficina & 263.68 & 100 & 263.68 & & 0 \\
Activos intangibles & 2929.79 & 100 & 2929.79 & & 0 \\
Capital de trabajo & 14695.40 & 100 & 14695.40 & & 0 \\
\hline TOTAL & $\mathbf{8 5 1 6 0 . 4 5}$ & $\mathbf{2 8 . 8 4}$ & $\mathbf{2 4 5 5 7 . 5 7}$ & $\mathbf{7 1 . 1 6}$ & $\mathbf{6 0 6 0 2 . 8 8}$ \\
\hline
\end{tabular}

Tabla 3. Costos Totales y unitarios en USD estimados para 10 años de duración del proyecto de venta de muebles de cashavara por los pobladores de Jenaro Herrera a los Estados Unidos.

$\begin{array}{lllllllllll}\text { Detalle Año } 1 & \text { Año } 2 & \text { Año } 3 & \text { Año } 4 & \text { Año } 5 & \text { Año } 6 & \text { Año } 7 & \text { Año } 8 & \text { Año } 9 & \text { Año } 10\end{array}$

\begin{tabular}{lcccccccccc}
\hline Total costos y gastos & 167001 & 170099 & 173025 & 175755 & 178264 & 174078 & 178775 & 183471 & 188175 & 192871 \\
\hline Oferta anual & 300 & 300 & 300 & 300 & 300 & 300 & 300 & 300 & 300 & 300 \\
\hline Costo unitario & 556 & 566 & 576 & 585 & 594 & 580 & 595 & 611 & 627 & 642 \\
\hline
\end{tabular}


Tabla 4. Descripción del Período de Recuperación de la Inversión para la elaboración de muebles con fibra de cashavara por pobladores de Jenaro Herrera.

\begin{tabular}{cccc}
\hline Años & FNC & FNCA & FNCAA \\
\hline 0 & 85160.45 & 85160.45 & -85160.45 \\
1 & 46894.11 & 41261.87 & -43898.58 \\
2 & 46039.68 & 35644.57 & -8254.01 \\
$\mathbf{3}$ & $\mathbf{4 5 1 5 2 . 1 3}$ & $\mathbf{3 0 7 5 8 . 8 4}$ & $\mathbf{- 2 2 5 0 4 . 8 3}$ \\
4 & 44226.97 & 26509.98 & 49014.82 \\
\hline 5 & 43259.05 & 22815.49 & 71830.31 \\
6 & 57581.97 & 26722.06 & 98552.36 \\
7 & 56696.60 & 23151.06 & 121703.43 \\
8 & 55811.22 & 20052.38 & 141755.81 \\
\hline 9 & 54920.17 & 17362.29 & 159118.10 \\
10 & 87377.24 & 24305.46 & 183423.56 \\
\hline & & & \\
\hline
\end{tabular}

\section{DISCUSIONES}

El mercado seleccionado para el estudio fue el estadounidense. Este mercado es de mayor interés al presentar la mayor demanda por muebles con fibras naturales, $28 \%$ de la demanda internacional o una inversión en importaciones de US\$ 612374 en el último año (POM EEUU, 2007; ITC, 2015).

Cabe destacar que el Perú tiene una ventaja con EE.UU., pues ambos países poseen un tratado de libre comercio (TLC) el cual exonera de aranceles a este tipo de muebles.

Según lo encontrado en Trademap, y lo presentado por Intriago (2008), se puede observar que los precios de los muebles de fibras naturales se encuentran en alza, siendo un momento propicio para su comercialización.

El tamaño del proyecto será de 300 juegos de sala anuales, considerando que cada artesano puede elaborar dos juegos de sala por mes, valor mínimo mencionado por los encuestados.

Comparando los resultados obtenidos en la evaluación financiera de Plazarte (2010), se puede apreciar que el VAN obtenido para muebles con esterillados de fibra de cashavara es superior al VAN obtenido para muebles con esterillado de fibra de mimbre.

Esta gran diferencia se debe a que en ese proyecto se contempló una gran inversión en la compra de muebles, generando mayores gastos. Por el contrario, en el proyecto de muebles con esterillado de cashavara se plantea la fabricación propia de los muebles, el tejido y acabado, lo cual reduce los costos permitiendo obtener una mayor ganancia en la utilidad.

El rendimiento obtenido por la comercialización de muebles de cashavara es superior al porcentaje mínimo exigido por la tasa de descuento, debido a que esta tasa exige un rendimiento del $13.64 \%$ y en la TIR se aprecia que se obtendrá el 55\%, es decir, 41 puntos sobre lo requerido.

Comparando con el rendimiento de Plazarte (2010), el cual solo se aleja en 5\% de su tasa de descuento, el proyecto de muebles de cashavara es mucho más rentable por ser un sistema más complejo que abarca desde la producción hasta la comercialización, reduciendo costos y aumentando los beneficios.

Con respecto a la rentabilidad sobre el capital propio, el proyecto alcanzará el $190.71 \%$ de rentabilidad sobre el capital aportado por los accionistas, a diferencia de Plazarte (2010), quien solo consigue $47.25 \%$ de rentabilidad sobre el capital propio y con una recuperación en 10 años. Esta diferencia determina que se produzcan mayores ganancias para los accionistas (asociación de artesanos de Jenaro Herrera), los cuales recuperarán la inversión en solo tres años. 
Apéndice 1. Descripción del Estado de Situación Inicial para la comercialización de muebles con esterillado de fibra de cashavara por los pobladores de Jenaro Herrera.

\begin{tabular}{|c|c|c|c|c|c|}
\hline \multicolumn{3}{|c|}{ Activo } & \multicolumn{3}{|c|}{ Pasivo } \\
\hline Activo corriente & & 14695.4 & Pasivo a largo plazo & & 60602.88 \\
\hline Capital de trabajo & 14695.4 & & Préstamo a largo plazo & 60602.88 & \\
\hline Activo fijo & & 67535.26 & Total pasivo & & \\
\hline Edifico & 17754.51 & & & & \\
\hline Terreno & 7910.42 & & & & \\
\hline Vehículo & 633868.34 & & & & \\
\hline Maquinaria y equipo taller & 3867.009 & & Patrimonio & & 24557.57 \\
\hline Maquinaria y equipo bodega & 292.9787 & & Capital & 24557.5676 & \\
\hline Muebles y enseres & 1439.111 & & & & \\
\hline Equipos de computación & 2139.202 & & & & \\
\hline Equipos de oficina & 263.6809 & & & & \\
\hline Activos diferidos intangibles & & 2929.79 & & & \\
\hline Total activos & & 85160.45 & Total pasivos + patrimonio & & 85160.45 \\
\hline
\end{tabular}

\section{CONCLUSIONES}

El sector artesanal en Perú tiene un potencial de comercialización que debe ser explotado y promocionado. Este estudio contribuye a demostrar que es posible generar negocios con productos forestales no maderables para exportación. Los análisis y evaluaciones realizadas determinaron que es factible exportar muebles con esterillado de fibra de cashavara producidos por una asociación de artesanos de Jenaro Herrera a los Estados Unidos, pues este país posee la mayor demanda insatisfecha por muebles de fibras naturales a nivel internacional. Una ventaja adicional es el tratado de libre comercio (TLC) que existe entre los Estados Unidos y el Perú, el cual exonera de aranceles a este tipo de muebles.

\section{BIBLIOGRAFÍA CITADA}

Balslev, H.; Grandez, C.; Paniagua, N.; Moller, A.; Lykke, S. 2008. Palmas (Arecaceae) útiles en los alrededores de Iquitos, Amazonía Peruana. Revista peruana de biología. 15(1): 121- 132.

Baluarte, J. 2000. La manufactura de muebles a partir de productos forestales no maderables en Iquitos-Perú. Folia Amazónica 11:(1-2).

Beltran, A; Cueva, H. Evaluación Privada de Proyectos. Segunda Edición. Universidad del Pacífico, Lima. 712 pp.
Bjorholm S.; Svenning, J.; Skov, F.; Balslev, H. 2005. Environmental and spatial controls of palm (Arecaceae) species richness across the Americas. Global Ecology and Biogeography 14: 423-429.

Carneiro, R. 2006. Palmeiras (Arecaceae) das Restingas do Estado do Rio de Janeiro, Brasil. Acta bot. bras. 20(3): 501-512.

Coral, C. 2009. Estudio de factibilidad para la Implementación de un taller de manufactura de muebles de estructura metálica básica de Uso doméstico. Tesis de pregrado, Escuela Superior Politécnica de Chimborazo, Facultad de mecánica, Riobamba,Ecuador. 93 pp.

Gallego, L. 2005. El tejido en chambira, una actividad que une más que sogas. Boletín de Antropología Universidad de Antiquia 19(36): 164-185.

Guevara, L.; Garzón, P. 2008. Morfoanatomía de órganos vegetativos aéreos en Desmoncus orthacanthos Mart. (Arecaceae, Arecoideae). ERNSTIA 18(1): 71-88

Henderson A.; Chavez, F. 1993. Desmoncus as a useful palm in the western Amazon. Principes 34: 184-186.

Henderson, A.; Galeano, G.; Bernal, R. 1995. Field Guide to the palms of the Americas. Princeton. University Press. Princeton, New Jersey. 199pp. 
Hübschmann, L.; Kvist, L.; Grandez, C.; Balslev, H. 2007. Uses of Vara Casha - a Neotropical Liana Palm, Desmoncus polyacanthos - in Iquitos, Peru. Palms 51(4): 167-176.

Intriago, P. 2008. Proyecto para la Utilización de la Fibra de Abacá en la Elaboración de Muebles Artesanales en la Ciudad de Santo Domingo para su Consumo en la Ciudad de Guayaquil. Guayaquil, Ecuador.

Llacsahuanga, D.; Venancio, M. 2011. Estudio de prefactibilidad para la instalación de una planta procesadora de goma de sapote (Capparis scabrida) con fines de exportación al mercado europeo. Tesis de pregrado, Universidad Nacional Agraria "La Molina", Facultad de Ciencias Forestales, Lima, Perú. 260 pp.

Mansur, E. 1995. El mimbre, una pequeña industria prometedora. Bosques y Desarrollo 14:38-40.

Marshall, A. 1890. Principles of Economics: A bibliographical Note. The Economical Journal 52:290-300.

Meza, C.; Sabogal. C.; de Jong, W. 2006. Rehabilitación de áreas degradadas en la Amazonia peruana : Revisión de experiencias y lecciones aprendidas. Bogor, Indonesia, CIFOR. Country case studies on Review of Forest Rehabilitation Initiatives: Lessons from the Past. $106 \mathrm{pp}$.

MINPRODUCE. 2013. Anuario Estadístico Industrial, Mipyme y Comercio Interno 2012. Perú. 138 pp.

FAO. 1990. Pequeñas empresas de elaboración de productos del bosque. Roma. 290 pp.

Plazarte, V. 2010. Proyecto de factibilidad para la elaboración de muebles de mimbre. Tesis de pregrado, Universidad Tecnológica Equinoccial. Facultad de Ciencias Económicas y Negocios, Quito, Ecuador $171 \mathrm{pp}$.

Tagle, X. 2014. Informe diagnóstico: El mercado de fibra de cashavara (Desmoncus polyacanthos) en la región Loreto. PROBOSQUES - IIAP. Iquitos. Perú 32 pp.

Torres, G. 2006. Evaluación biométrica en plantaciones de desmoncus polyacanthos Martius "cashavara" en los ecosistemas aluviales de Jenaro Herrera, Loreto, Perú.

Torres, G.; Delgado, C.; Mejía, K. 2010. Potential harvest of Desmoncus polyacanthos (Arecaceae) in the Peruvian amazon. Palms, 54(2):73-76.

Torres, G.; Rodríguez, E.; Delgado, C.; Del Castillo, D. 2009. Cosecha de cashavara Desmoncus polyacanthos Martius, en los bosques inundables de la amazonía peruana. Folia Amazónica. 18(1): $51-57$.

Vásquez, M. 1994. Extracción y comercialización de productos forestales diferentes de la madera en el ámbito de la Unidad Forestal de Iquitos . $135 \mathrm{pp}$.

Vela, C. 2013. Evaluación de la manufactura y comercialización de artesanías de "Chambira" Astrocaryum chambira, en las comunidades nativas San Antonio y Atalaya, cuenca del Nanay. Tesis de pregrado, Universidad Nacional de la Amazonía Peruana, Facultad de Ciencias Forestales, Iquitos, Perú. 51 pp.

Recibido: 14 de Marzo del 2016

Aceptado para publicación: 18 de Abril del 2016 
